\title{
Laminar mixed convection heat transfer of SiC-EG nanofluids in a triangular enclosure with a rotating inner cylinder: simulations based on the measured thermal conductivity and viscosity"
}

\author{
Yu-fei WANG ${ }^{1,2}, \mathrm{Xu} \mathrm{XU}^{\dagger+1}$, Tian TIAN ${ }^{3}$, Li-wu FAN ${ }^{2}$, Wen-long WANG ${ }^{1}$, Zi-tao $\mathrm{YU}^{2}$ \\ $\left({ }^{1}\right.$ College of Metrological and Measurement Engineering, China Jiliang University, Hangzhou 310018, China) \\ ('Institute of Thermal Science and Power Systems, Department of Energy Engineering, Zhejiang University, Hangzhou 310027, China) \\ $\left({ }^{3}\right.$ Department of Mechanical, Materials, and Aerospace Engineering, University of Central Florida, Orlando, FL 32816, USA) \\ †E-mail: xuxu@cjlu.edu.cn
}

Received May 5, 2014; Revision accepted Nov. 18, 2014; Crosschecked May 7, 2015

\begin{abstract}
A numerical study has been carried out for a laminar steady mixed convection flow in a 2D triangular enclosure with an inner rotating coaxial cylinder, with the enclosure filled with ethylene glycol-silicon carbide (SiC-EG). The thermal conductivity and viscosity of the SiC-EG nanofluids were experimentally determined by using a Decagon Devices KD2 Pro thermal property meter and a rotational Brookfield viscometer, respectively. Various pertinent parameters, such as the dimensionless rotation velocity, solid volume fraction, dimensionless radius of the inner cylinder, and Rayleigh numbers, were analyzed to determine their influences on heat transfer and fluid flow. Results clearly show how the direction of rotation of the cylinder affects the thermal performance in a triangular enclosure. It is found that the average Nusselt number increases with rise in the Rayleigh number or as more nanoparticles are added to the base liquid. It was also observed that at constant Rayleigh number, different rotational conditions have remarkable effects on the flow and heat transfer characteristics.
\end{abstract}

Key words: Ethylene glycol-silicon carbide (SiC-EG) nanofluids, Mixed convection, Triangular enclosure, Rotating cylinder, Rayleigh number

doi:10.1631/jzus.A1400120

Document code: A

CLC number: TK124

\section{Introduction}

Problems of convective flow and heat transfer in an enclosure with a coaxial stationary cylinder have been studied extensively (Kuehn and Goldstein, 1976; Kim et al., 2008; Lee et al., 2010; Xu et al., 2010; 2012; Yu et al., 2010; Bararnia et al., 2011; Parvin et $a l ., 2012$ ), with some special attention to the analysis of laminar convection inside a triangular closed cavity (Xu et al., 2010; 2012; Yu et al., 2010) since such a

\footnotetext{
${ }^{\star}$ Corresponding author

* Project supported by the National Natural Science Foundation of China (No. 51106144)

(D) ORCID: Yu-fei WANG, http://orcid.org/0000-0002-9449-8705; Xu XU, http://orcid.org/0000-0003-3697-2787

(C) Zhejiang University and Springer-Verlag Berlin Heidelberg 2015
}

geometry often has engineering applications. Xu et al. (2010) numerically investigated the laminar steady natural convection in an air-filled triangular cavity with a motionless circular cylinder inside. The convective heat transfer of a low-Prandtl-number fluid from a heated horizontal circular cylinder to its coaxial triangular enclosure was documented by Yu et al. (2010) and $\mathrm{Xu}$ et al. (2012).

A spectral element method (Ghaddar and Thiele, 1994) was adopted to study the problems of mixed convection in an isothermal rectangular enclosure; then $\mathrm{Fu}$ et al. (1994) employed a penalty finite element numerical method to investigate the enhancement of natural convection heat transfer in an enclosure with a rotating circular cylinder. Ghaddar and Thiele (1994) and $\mathrm{Fu}$ et al. (1994) found the heat 
transfer was enhanced by the presence of a rotating body. In fact, utilizing the rotation of a cylinder inside to enhance heat transfer in enclosures has become a promising approach. Additionally, the fluid flow and heat transfer around a rotating cylinder are considered to be fundamental problems in fluid mechanics with many practical applications, such as nuclear reactor fuel rods, drilling of oil wells, rotating-tube heat exchangers, rotating shafts, and steel suspension bridge cables. However, there has been less work involving rotating cylinders compared with that on stationary cylinders. Costa and Raimundo (2010) analyzed the flow structure, temperature field, and heat transfer process by using dimensionless stream function, temperature, and heat function. The effects of rotating heated cylinders with different geometric cross sections in rectangular enclosures were investigated by Ghaddar (1996) and Shih et al. (2009). Not surprisingly, the rotational direction of the cylinder has significant effects on convection heat transfer (Ghaddar, 1996; Costa and Raimundo, 2010; Ghasemi and Aminossadati, 2010; Liao and Lin, 2012). Moreover, Misirlioglu (2006) concluded that enhancement of heat transfer could be obtained when forced convection started to be significant.

Since the previous works mostly used traditional heat transfer fluids, such as air, water, and ethylene glycol (EG) that usually have poor heat transfer performance, Choi and Eastman (1995) suggested using fluids containing nano-sized solid particles with high thermal conductivity to enhance their thermal performance; these are referred to as nanofluids. Recently, Roslan et al. (2012) studied the effects on heat transfer of a rotating circular cylinder in a square enclosure filled with different kinds of nanofluids. They drew the conclusion that the strength of flow circulation and heat transfer was much stronger for a higher nanoparticle concentration with better conductivity.

In an effort to extend the existing knowledge, the main concern of this paper is to study the mixed convection problem with a circular coaxial cylinder in a triangular enclosure filled with ethylene glycolsilicon carbide (SiC-EG) nanofluids. The thermal conductivity and the viscosity of SiC-EG nanofluids are experimentally measured and compared with the classic predictive model of the thermophysical prop- erties of low-volume fraction liquid-solid suspensions with randomly dispersed particles. The results in this study are hoped to be of value in the application of the rotational cylinder to enhance thermal performance in an enclosure filled with nanofluids.

\section{Problem description}

\subsection{Physical model and mathematical formulation}

The configuration of a 2D triangular enclosure, of both width and height $L$, with the rotating cylinder placed in the center of an inscribed circle of isosceles right triangle enclosure is sketched in Fig. 1. The left side wall and the opposite right side inclined wall are subjected to isothermal temperatures $T_{\mathrm{H}}$ and $T_{\mathrm{C}}$ respectively, assumed $T_{\mathrm{H}}>T_{\mathrm{C}}$. The bottom of the enclosure is assumed perfectly insulated. The triangular enclosure is filled with SiC-EG nanofluids and the gravitational acceleration $(g)$ is assumed to be acting downwards, i.e., the negative $y$ direction. Under the influence of the vertical gravitational field, the hot and cold surfaces at different temperatures lead to a natural convection problem. Due to the non-slip boundary condition for velocity on its surface, the rotating cylinder induces a forced flow. The overall situation results in a mixed convection problem based on the rotating speed of the cylinder. The maximum and the minimum values of the thermal conductivity of the inner cylinder correspond to isothermal and adiabatic boundary conditions, respectively. Considering general applications where the thermal conductivity of the inner rotating cylinder is relatively low, this study assumes that the surface of inner cylinder is kept insulated.

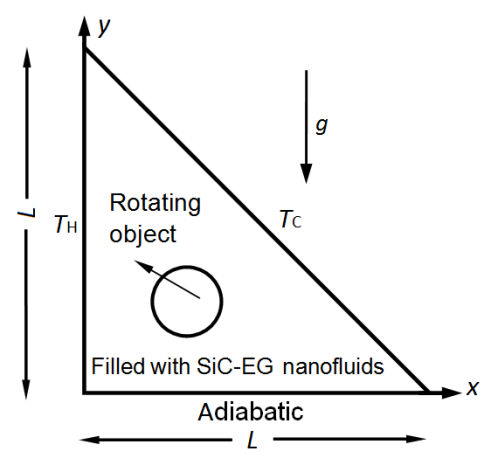

Fig. 1 Schematic diagram of the physical model 


\subsection{Thermophyiscal properties of nanofluids}

The nanofluids for this study were prepared by mixing EG with a water-based suspension of $\mathrm{SiC}$ nanoparticles. The volume fractions of nanoparticles formulated in this experiment varied in the range between 0 and 0.04 , and the pure fluid used in this experiment was a mixture of EG $(60 \%)$ and water $(40 \%)$ by weight. The mixture model used to predict the effective density $\rho$ of the nanofluids is given by

$$
\rho_{\mathrm{nf}}=(1-\varphi) \rho_{\mathrm{f}}+\varphi \rho_{\mathrm{np}}
$$

the effective heat capacitance of the nanofluids is expressed as

$$
\left(\rho C_{\mathrm{p}}\right)_{\mathrm{nf}}=(1-\varphi)\left(\rho C_{\mathrm{p}}\right)_{\mathrm{f}}+\varphi\left(\rho C_{\mathrm{p}}\right)_{\mathrm{np}}
$$

and the thermal expansion coefficient of the nanofluids can be determined by

$$
\beta_{\mathrm{nf}}=(1-\varphi) \beta_{\mathrm{f}}+\varphi \beta_{\mathrm{np}}
$$

where $C_{\mathrm{p}}$ is the specific thermal capacity, $\varphi$ is the solid volume fraction, and $\beta$ is the thermal diffusivity. The subscripts $\mathrm{f}$, nf, and $\mathrm{np}$ represent pure fluid, nanofluid, and nanoparticle, respectively.

Unlike other research where the thermal conductivity and viscosity of nanofluids were determined by existing models, in this study these two important thermal properties were, because of their unpredictability, measured experimentally. The SiC nanoparticles (Shanghai Super Power Kano Co. Ltd., China) were of average diameter $40 \mathrm{~nm}$ and spherical shape. The nanofluids were prepared following a two-step protocol. Thermal conductivity of the SiC-EG nanofluids was obtained by Decagon Devices KD2 Pro thermal property meter (Decagon Devices, USA) based on the traditional transient hot-wire method. The viscosity of the SiC-EG nanofluids was measured using a rotational Brookfield viscometer. During the experiment, the temperature of the $\mathrm{SiC}$-EG nanofluids was kept at $20{ }^{\circ} \mathrm{C}$. The experimental results are compared with well-established models and listed in Table 1. Basically, the experimental results are consistent with those of the theoretical models, and some deviation can possibly be attributed to differences in a few specific factors, such as temperature, nanoclusters, Brownian motion, $\mathrm{pH}$ of nanofluids, and thermophoresis.

Table 1 Comparison of the thermal conductivity $(k)$ and viscosity $(\mu)$ between the values calculated by the Maxwell and Brinkman models and the experimental results for EG-SiC nanofluids as a function of SiC volume fractions

\begin{tabular}{lccccc}
\hline & \multicolumn{2}{c}{$k(\mathrm{~W} /(\mathrm{m} \cdot \mathrm{K}))$} & & \multicolumn{2}{c}{$\mu(\mathrm{kg} /(\mathrm{m} \cdot \mathrm{s}))$} \\
\cline { 2 - 3 } \cline { 5 - 6 }$\varphi$ & $\begin{array}{c}\text { Experi- } \\
\text { mental }\end{array}$ & \begin{tabular}{c} 
(Maxwell, \\
\cline { 5 - 6 }
\end{tabular} & & $\begin{array}{c}\text { Experi- } \\
\text { mental }\end{array}$ & $\begin{array}{c}\text { (Brinkman, } \\
1952)\end{array}$ \\
\hline 0 & 0.2510 & 0.2510 & & 0.02056 & 0.02056 \\
0.01 & 0.2574 & 0.2637 & & 0.02100 & 0.02108 \\
0.02 & 0.2629 & 0.2766 & & 0.02182 & 0.02163 \\
0.03 & 0.2786 & 0.2898 & & 0.02358 & 0.02219 \\
0.04 & 0.3061 & 0.3032 & & 0.02898 & 0.02277 \\
\hline
\end{tabular}

\section{Computational methodology}

\subsection{Governing equations}

A 2D, steady state, incompressible, laminar flow model is considered in the present study. The governing equations are solved by using the controlvolume-based computational fluid dynamics (CFD) code Fluent (version 6.3), and a pressure-based solver is adopted. Furthermore, to improve numerical accuracy, the third-order correction quadratic upwind interpolation of convective kinematics (QUICK) scheme and second-order central difference are employed for the convection and the diffusion terms. The computational domain (Fig. 1) was created in the environment of the preprocessing code Gambit (version 2.4). The SIMPLEC algorithm was employed to couple velocity and pressure fields. In addition, the absolute convergence criteria are set to be $10^{-4}$ and $10^{-6}$ for velocities and energy, respectively. It is assumed that the fluid properties are constant, except for the density in the buoyancy term, following the Boussinesq approximation. So the non-dimensional governing equations under the Boussinesq approximation are written in general form as follows (Ghasemi and Aminossadati, 2010):

continuity: $\quad \frac{\partial U}{\partial X}+\frac{\partial V}{\partial Y}=0$,

$X$-momentum: 
$U \frac{\partial U}{\partial X}+V \frac{\partial V}{\partial Y}=\frac{\partial}{\partial X}\left(\frac{\mu_{\mathrm{nf}}}{\rho_{\mathrm{nf}} \alpha_{\mathrm{f}}} \frac{\partial U}{\partial X}\right)+\frac{\partial}{\partial Y}\left(\frac{\mu_{\mathrm{nf}}}{\rho_{\mathrm{nf}} \alpha_{\mathrm{f}}} \frac{\partial U}{\partial Y}\right)-\frac{\partial P}{\partial X}$

$Y$-momentum:

$$
\begin{aligned}
U \frac{\partial U}{\partial X}+V \frac{\partial V}{\partial Y}= & \frac{\partial}{\partial X}\left(\frac{\mu_{\mathrm{nf}}}{\rho_{\mathrm{nf}} \alpha_{\mathrm{f}}} \frac{\partial V}{\partial X}\right)+\frac{\partial}{\partial Y}\left(\frac{\mu_{\mathrm{nf}}}{\rho_{\mathrm{nf}} \alpha_{\mathrm{f}}} \frac{\partial V}{\partial Y}\right) \\
& -\frac{\partial P}{\partial Y}+\frac{(\rho \beta)_{\mathrm{nf}}}{\rho_{\mathrm{nf}} \beta_{\mathrm{f}}} \operatorname{RaPr} \theta,
\end{aligned}
$$

energy:

$$
U \frac{\partial \Theta}{\partial X}+V \frac{\partial \Theta}{\partial Y}=\frac{\partial}{\partial X}\left(\frac{\alpha_{\mathrm{nf}}}{\alpha_{\mathrm{f}}} \frac{\partial \Theta}{\partial X}\right)+\frac{\partial}{\partial Y}\left(\frac{\alpha_{\mathrm{nf}}}{\alpha_{\mathrm{f}}} \frac{\partial \Theta}{\partial Y}\right),
$$

where $\alpha$ and $\mu$ represent thermal diffusivity and dynamic viscosity, and $R a$ and $P r$ are the Rayleigh number and the Prandtl number, respectively.

The dimensionless parameters for the space coordinates, the velocity components, the temperature, and the driving pressure in Eqs. (4)-(7) are defined, respectively, as

$$
\begin{gathered}
X=\frac{x}{L}, \quad Y=\frac{y}{L}, \quad U=\frac{u L}{\alpha_{f}}, \quad V=\frac{v L}{\alpha_{f}}, \\
\Theta=\frac{\left(T-T_{\mathrm{c}}\right)}{\left(T_{\mathrm{h}}-T_{\mathrm{c}}\right)}, \quad P=\frac{(p+\rho g y) L^{2}}{\rho_{\mathrm{nf}} \alpha_{\mathrm{f}}^{2}},
\end{gathered}
$$

where $u$ and $v$ are the velocity components, and $p$ is the fluid pressure.

The Rayleigh number $(R a)$ and the Prandtl number $(P r)$ are defined in terms of fluid properties as

$$
R a=\frac{g \beta_{\mathrm{f}}\left(T_{\mathrm{H}}-T_{\mathrm{C}}\right) L^{3}}{v_{\mathrm{f}} \alpha_{\mathrm{f}}}, \quad \operatorname{Pr}=\frac{u_{\mathrm{f}}}{\rho_{\mathrm{f}} \alpha_{\mathrm{f}}}
$$

Evaluation of the relative intensity between natural and forced convection can be quantified by a modified non-dimensional parameter of the Richardson number (Liao and Lin, 2012), defined as $R i=R a /\left(\operatorname{Pr} \cdot \operatorname{Re}^{2}\right)$, where $R e$ is the Reynolds number $\left(R e=\rho \cdot \omega_{\text {cylinder }} \cdot r / \mu\right)$, and $\omega_{\text {cylinder }}$ is the surface rotational velocity of the inner cylinder and $r$ is the radius of the inner cylinder. For this study, this nondimensional parameter can be defined as (Costa and Raimundo, 2010)

$$
R i_{\mathrm{nf}}=\frac{R a_{\mathrm{nf}} P r_{\mathrm{nf}}}{4 \Omega^{2} R^{4}}
$$

where $R$ is the non-dimensional radius of the inner cylinder given as $R=r / L$, and $\Omega$ is the non-dimensional angular rotational velocity given as $\Omega=\omega L^{2} / \alpha_{\mathrm{f}}^{2}$, where $\omega$ represents the angular rotational velocity.

In general, the heat transfer regime can be divided into three parts according to the magnitude of the Richardson number: natural convection, mixed convection, and forced convection. In this study, mixed convection for the nanofluids can be achieved by imposing a rotating cylinder across the enclosure. Natural convection dominates when the value of $R i_{\mathrm{nf}}$ is relatively high, otherwise forced and mixed convection prevail.

Eq. (10) indicates that all following dimensionless parameters $R a, P r, \Omega$, and $R$ will be applied to identify the heat transfer regime. Since the only fluid concerned in this study is a SiC-EG nanofluid, the Prandtl number $(\mathrm{Pr})$ is fixed at approximately 7.0 throughout this study. Therefore, the Richardson number $(R i)$ is simply dependent on the Rayleigh number and the dimensionless rotational velocity. According to Costa and Raimundo (2010) and Roslan et al. (2012), it is assumed that in the case of $\Omega<0$ the inner cylinder moves in the same direction as the flow induced by natural convection from the temperature differences between the hot and cold surfaces. On the contrary, the cylinder revolves against the natural convection induced flow for $\Omega>0$. So different combinations of the natural and forced convections (combined or opposed) are considered.

It is assumed that (1) both the fluid and nanoparticles are in a thermal equilibrium state, (2) non-slip velocity condition applies on the interface between fluid and particles, and (3) the nanofluids in the enclosure are Newtonian, incompressible, and laminar. The corresponding boundary conditions of the entire computational domain for dimensionless velocity and temperature are taken as follows:

Along the left vertical wall of the triangle enclosure: $\Theta=1$;

Along the right inclined wall of the triangle enclosure: $\Theta=0$;

Along the horizontal wall of the triangle enclosure: $\frac{\partial \Theta}{\partial Y}=0$;

Along all the walls of the enclosure: $U=V=0$;

Along the inner rotating cylinder: $\frac{\partial \Theta}{\partial N}=0(N$ 
represents the normal direction of the inner rotating cylinder $),|\boldsymbol{V}|=|\Omega| \cdot R(|\boldsymbol{V}|$ represents the absolute value of velocity over the surface of the inner cylinder).

The physical quantities of interest are the local Nusselt number along the hot wall, $N u_{L}$, which is defined as

$$
N u_{L}=\frac{q L}{k\left(T_{\mathrm{H}}-T_{\mathrm{C}}\right)},
$$

where $q$ is the local heat flux. The surface-averaged Nusselt number $N u_{\mathrm{m}}$ is used to represent the overall heat transfer rate within the domain of interest. At steady-state, it is obvious that the average Nusselt numbers along both the hot and cold surfaces are identical. Hence, the average Nusselt number can be determined by integrating the local Nusselt number along the hot surface:

$$
N u_{\mathrm{m}}=\int_{0}^{1} N u_{L} \mathrm{~d} Y
$$

\subsection{Code validation}

A numerical procedure based upon a finite volume method is adopted. Although the commercial CFD code Fluent has been widely used in solving thermo-fluids problems, a validation test is still carried out to verify its application to this problem.

The present numerical simulation results are compared with the experimental results of Krane and Jessee (1983) for natural convection in an enclosure filled with air as shown in Fig. 2 and with the numerical work of Roslan et al. (2012) that considered

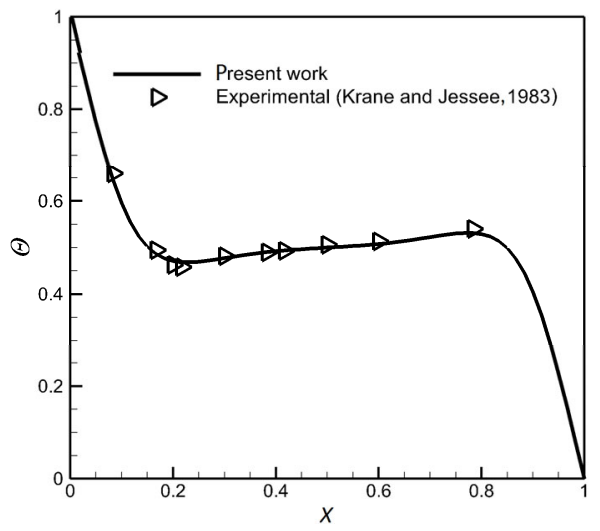

Fig. 2 Comparison of temperature distribution between present simulation and the experimental data of Krane and Jessee (1983) at $R a=1.89 \times 10^{5}, P r=0.71$ mixed convection induced by a rotating cylinder in a square enclosure as listed and shown in Table 2 and Fig. 3 in terms of mean Nusselt number and streamlines, respectively.

Table 2 Comparison of the mean Nusselt number between the present work and Roslan et al. (2012)'s result with respect to water-Cu nanofluids at $R a=10^{5}, R=0.2$, and $\varphi=0.05$ for three different dimensionless rotational velocities

\begin{tabular}{cccc}
\hline \multirow{2}{*}{$\Omega$} & \multicolumn{2}{c}{ Mean Nusselt number } & \multirow{2}{*}{$\begin{array}{c}\text { Error } \\
\text { ny }\end{array}$} \\
\cline { 2 - 3 } & Present study & Roslan et al. (2012) & $(\%)$ \\
\hline-500 & 4.7156 & 4.8310 & -2.3887 \\
0 & 4.9632 & 5.0198 & -1.1275 \\
500 & 4.5746 & 4.7197 & -3.0743 \\
\hline
\end{tabular}

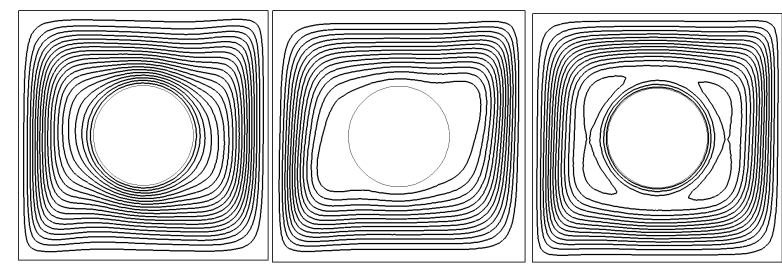

(a)

(b)

(c)

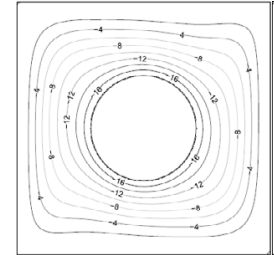

(d)

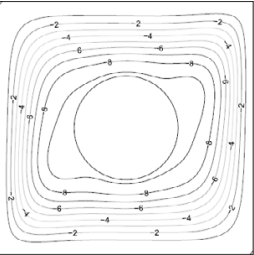

(e)

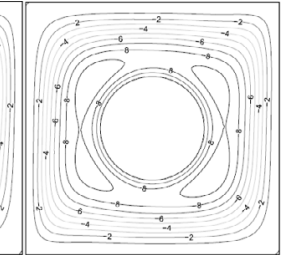

(f)
Fig. 3 Comparison of streamlines between the present work (a)-(c) and that of Roslan et al. (2012) (d)-(f) with respect to water-Cu nanofluids at $R a=10^{5}, R=0.2$, and $\varphi=0.05$ for $\Omega=-500$ (a) and (d), $\Omega=0$ (b) and (e), and $\Omega=500$ (c) and (f)

Figs. 3d-3f are reprinted from (Roslan et al., 2012), Copyright 2012, with permission from Elsevier

Note that the tiny differences from streamlines in Fig. 3 between the results of present work and Roslan et al. (2012) might be primarily attributed to the prediction of thermophysical properties of nanofluids using different models, in turn leading to deviations in determining the dimensionless rotation velocity and Rayleigh numbers. In this study, with the same condition ( $R=0.2, \varphi=0.05$ for water- $\mathrm{Cu}$ ), by employing the Maxwell (1904) and Brinkman (1952)'s models, the dimensionless angular velocity is calculated as 493.56, as against 500 in Roslan et al. (2012). In 
general, good agreement is achieved and thus the numerical method described may be used for the present simulations.

\subsection{Grid independence test}

A grid independency study for $\Omega=2000, R a=10^{5}$, $R=0.1$, and $\varphi=0.02$ has been carried out using unstructured triangular grids unevenly distributed over the computational domain and concentrated near the inner rotating cylinder. The present code was tested for grid independence by calculating both the average Nusselt number $N u_{\mathrm{m}}$ on the hot surface and the dimensionless maximum stream fuction $\psi_{\max }$ which are shown in Fig. 4 for six different grid sizes. It can be concluded from Fig. 4 that both the average Nusselt number and the maximum stream function are nearly independent of a grid density of 12066 cells which meets the requirements of the grid independency study. Therefore, considering the balance between numerical accuracy and the cost of computational time, a grid density of about 12066 cells is used for the present investigation.

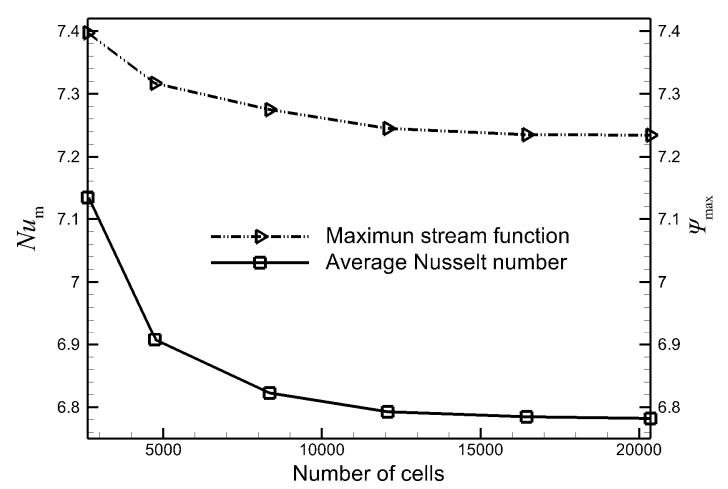

Fig. 4 Grid dependence study of Nusselt number and the maximum stream function for $\Omega=2000, R a=10^{5}, \varphi=0.02$, and $R=0.1$

\section{Results and discussion}

The present investigation was carried out with controlling parameters: (1) the dimensionless rotation velocity $\left(-5 \times 10^{3} \leq \Omega \leq 5 \times 10^{3}\right)$, (2) dimensionless radius $R(R=0.05,0.1,0.15$, and 0.2$)$, (3) the solid volume fraction $(\varphi=0,0.01,0.02,0.03$, and 0.04$)$, and (4) Rayleigh number $\left(R a=10^{4}, 10^{5}, 10^{6}\right.$, and $\left.10^{7}\right)$. As result of these ranges of dimensionless parameters, the values of the Richardson number ranges from 0.4375 to $\infty$. Then, a natural convection situation occurs when $\Omega$ is zero and mixed convection can be considered when $|\Omega|$ is greater than zero in the present study. The thermal performances of SiC-EG nanofluids with different rotating conditions of the inner cylinder were demonstrated and are discussed first. Special attention was then turned to the effects of the solid volume fraction for different Rayleigh numbers at a fixed non-dimensional rotational velocity of the inner cylinder.

\subsection{Effects of the rotation speed and the dimen- sionless radius of the inner cylinder}

Hydrodynamic and thermal behaviors of the SiC-EG nanofluids are visualized with the aid of streamlines and isotherms at various dimensionless rotation velocities of the inner cylinder for different dimensionless radii ranging from 0.05 to 0.2 . Special emphasis is given to the overall thermal performance of the left hot surface, which can be improved or weakened dependent on the magnitude and direction of the rotational velocity of the inner cylinder. In this section, $R a=10^{5}$ is selected because Ghaddar and Thiele (1994) stated that the rotation of an isoflux circular cylinder was able to enhance the heat transfer in the enclosure compared with the case of a motionless cylinder as $R a<2.7 \times 10^{5}$.

\subsubsection{Flow and temperature fields}

Comparisons of the flow fields for the rotating cylinder with different cross-section geometries are exhibited by means of streamlines in each column of Fig. 5. Generally, in the case of $\Omega<0$, the cylinder moves in the direction which is exactly the same as that of the flow aroused by natural convection due to the temperature differences between the left and right walls. That is, clockwise rotation of the cylinder induces an overall convective flow in the clockwise direction. For anti-clockwise rotation of the cylinder it spins against the flow direction of natural convection. Thus, the contribution of the inner rotating cylinder to natural convection flow and heat transfer strongly depends on whether its rotational direction is clockwise or anti-clockwise.

As for the negative rotational direction $(\Omega<0)$, four vortices are formed for the highest $R$ due to the small angled corners, the flow squeezes in that part, 
and the rotating direction of the cylinder being opposite to that of the natural convection flow. Also, the effect of forced convection increases. At that moment, the shape of streamlines is mainly conditioned by the triangular enclosure surface. With respect to the positive rotation direction $(\Omega>0)$, the rotating cylinder has a significant effect on the flow pattern. As it can be seen, it changes the flow patterns from natural convection to mixed convection. Note that among flow patterns (Figs. 5a-5d), only the inner cylinder with dimensionless radius $R=0.05$ basically keeps the characteristics of natural convection where the effect of forced convection is insignificant. It is clearly indicated that streamlines close to the inner cylinder get denser as the dimensionless radius increases. That indicates the fluid flow is intense here, while the streamlines become more densely packed next to the walls of the triangular enclosure as the dimensionless radius decreases.

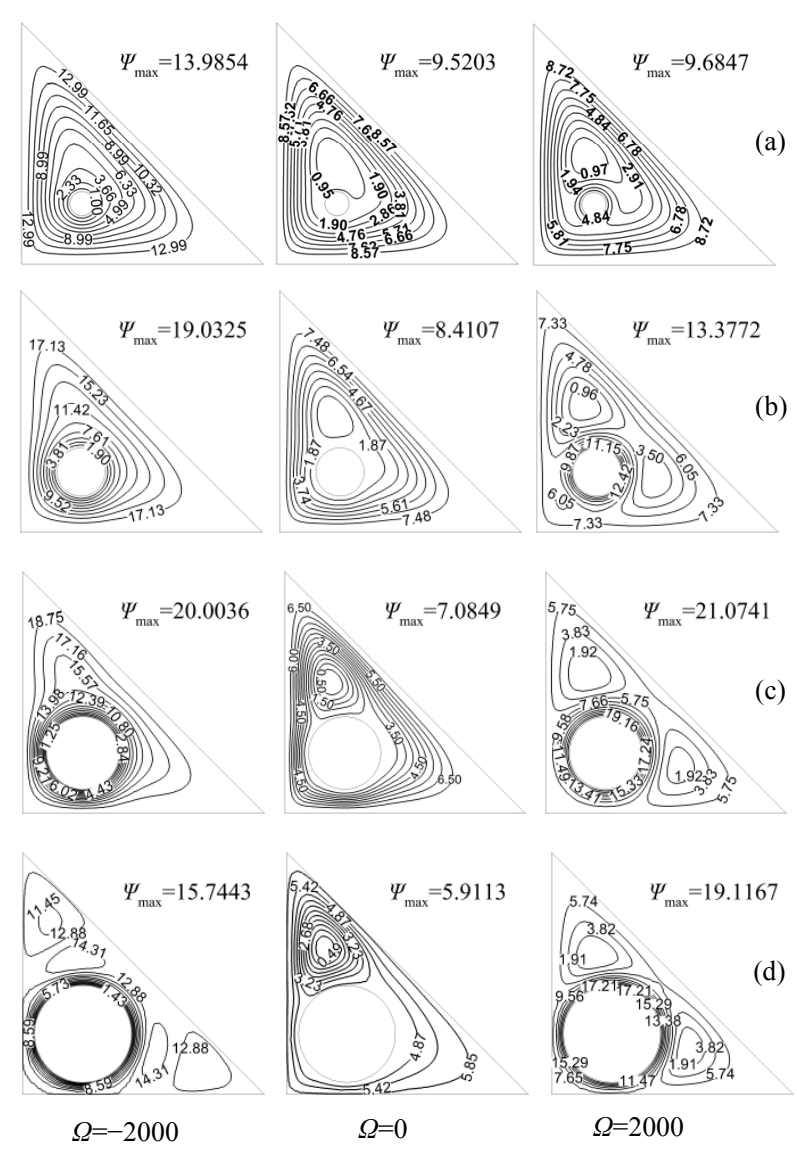

Fig. 5 Streamlines with respect to clockwise and anticlockwise directions and different dimensionless rotational velocities at $R a=10^{5}$ and $\varphi=0.02$ for $R=0.05$ (a), $R=0.1$ (b), $R=0.15$ (c), and $R=0.2$ (d)
To acquire a better understanding of the streamlines in Fig. 5, the intensity of flow circulation is examined and quantified by the maximum value of the dimensionless stream function $\Psi_{\max }$ which indicates the intensity of circulation flow. It gradually increases as the dimensionless radius of the cylinder increases from 0.05 to 0.15 and the maximum value occurs at $R=0.15$ for all the rotating conditions, but always decreases at $R=0.2$ because the radius of the cylinder is too large and the space for fluid flow between the cylinder and the enclosure walls becomes narrow and it is restrained. In the case $\Omega=-2000$, the forced and free convection flows are combined. That is, the flow induced by the inner cylinder works with the natural convection so that the buoyancy-driven convection is enhanced. Accordingly, the maximum value of the dimensionless stream of function of $\Omega=-2000$ apparently increases more than that of $\Omega=2000$ at $R=0.05$ where the effect of force convection is insignificant. This is mainly because the rotation of the inner cylinder accelerates the mass transfer in the cavity. The case $\Omega=0$ corresponds to a motionless cylinder which is presented in the middle column of Fig. 5. In this case, the fluid flow is due to natural convection only since the adiabatic stationary cylinder does not interact with the mass transferred by the fluid, so the case of $R=0.05$ has the greatest dimensionless maximum stream function due to the fact that the space for the fluid flow between the inner cylinder and the enclosure walls is the largest for natural convection circulation. It is worth noting that the increment of fluid flow area also enhances the viscous friction that tends to decrease the flow intensity, which was also indicated by Moukalled and Acharya (1996). However, the enhancement of the flow intensity resulting from natural convection is compensated for by the attenuation due to additional viscous friction.

\subsubsection{Heat transfer}

The dependence of isotherms on clockwise/ anti-clockwise direction or different dimensionless rotational velocities is presented in Fig. 6. As the dimensionless radius increases, the isotherms become more distorted and the thermal boundary layer starts to separate from the surface of the inner cylinder. At the fixed parameters, isotherms at the upper part of the hot surface are always comparatively accumulated, 
which implies a high thermal gradient there. Correspondingly, it has a similar trend and a peak value around the top of the hot surface for all dimensionless radii in that flow moves up along the hot wall of the triangular enclosure and impinges on the inclined cold walls and separates. In addition, because of the separation of the thermal boundary from the surface near the left side of the inner rotating cylinder, the nanofluid flow at the case of $\Omega=-2000$ gives the strongest impingement on the lower part of the hot surface, which enhances the heat transfer in this region $(Y=0.25)$. However, in the case of $\Omega=0$, a maximum value is obtained near the bottom wall of the left wall $(Y=0.1)$ and gradually descends with ascending value of $Y$, because the temperature difference between the wall and the fluid at the bottom gradually decreases. The case of $\Omega=2000$ has its maximum values at around $Y=0.35$ due to impingement of the flow resulting from the rotating cylinder which can be seen from the $N u_{L}$ in Fig. 7 . On the

(a)

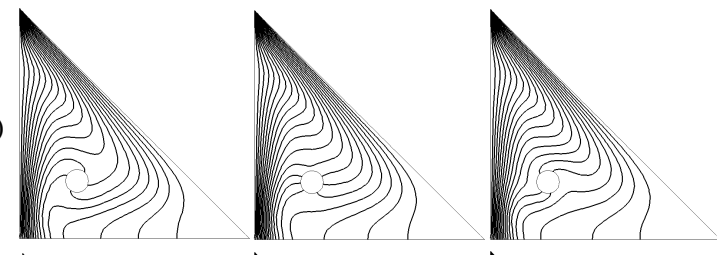

(b)

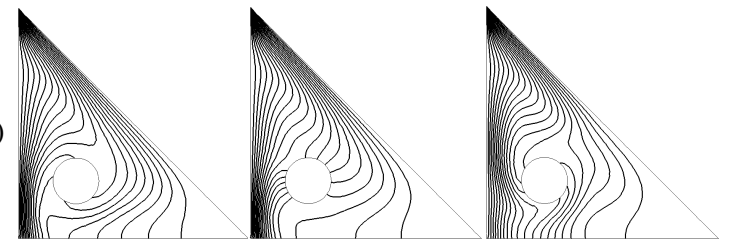

(c)

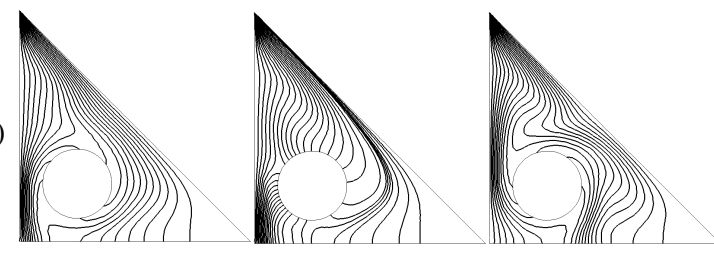

(d)

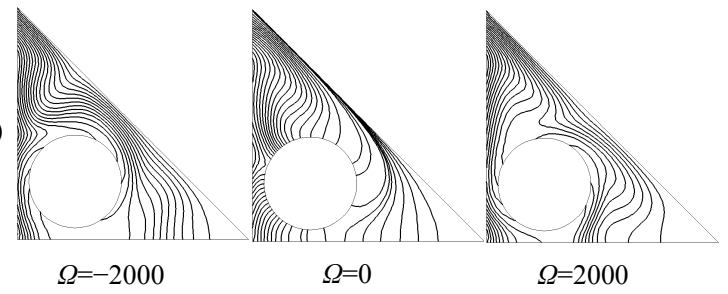

Fig. 6 Isotherms with respect to clockwise and anticlockwise direction and different dimensionless rotational velocities at $R a=10^{5}$ and $\varphi=0.02$ for $R=0.05$ (a), $R=0.1$ (b), $R=0.15$ (c), and $R=0.2$ (d)

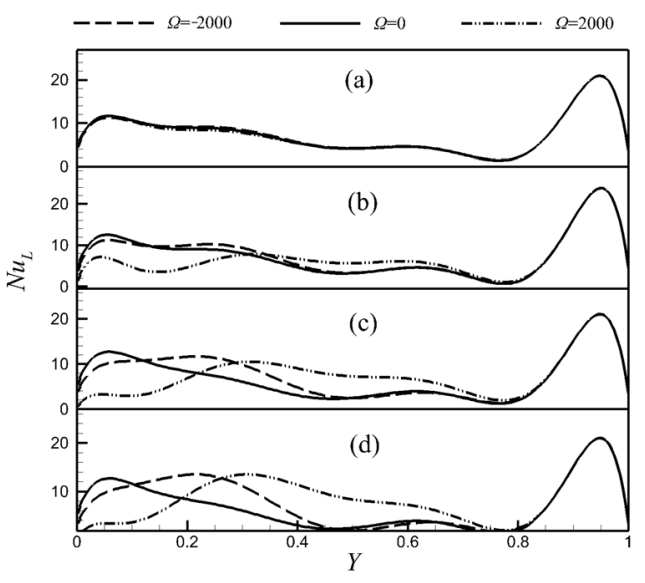

Fig. 7 Comparisons of local Nusselt number distribution along the hot surface with respect to different rotational velocities at $R a=10^{5}$ and $\varphi=0.02$ for $R=0.05$ (a), $R=0.1$ (b), $R=0.15$ (c), and $R=0.2$ (d)

other hand, the influence of the inner cylinder on the local Nusselt number becomes weaker as $R$ decreases, leading to the gap of the local Nusselt number profiles among different rotational speed getting narrower. Particularly, at $R=0.05$, the dimensionless rotational speed of the inner cylinder seems to have a negligible effect on the distribution of the local Nusselt numbers of the hot surface.

The average Nusselt number along the left side of the triangular enclosure is shown in Fig. 8 for different dimensionless rotational velocities. It can be seen that the average Nusselt number is closely dependent on the dimensionless rotational velocity. At a fixed radius, the average Nusselt number for a cylinder revolving at a negative rotational velocity is always higher than that of a motionless inner cylinder and the fastest negative rotation of inner cylinder at $\Omega=-5000$ gives the maximum average Nusselt number. This is due to the fact that the inner cylinder revolving at a negative dimensionless rotation speed significantly accelerates the main flow, which leads to a better heat transfer performance crossing the triangular enclosure. Besides, according to Eq. (10), the Richardson number will decrease when the dimensionless rotation velocity increases, which corresponds to enhanced forced convection. Hence, for high rotational velocities forced convection plays a significant role in the thermal characteristics about which similar findings have been presented in previous studies (Misirlioglu, 2006; Costa and Raimundo, 2010). By comparison, as the rotation direction goes 
opposite to natural convection $(\Omega>0)$, the heat transfer rate decreases at lower positive rotation speed for $R=0.05$ and 0.1 . In these situations, the cylinder rotation dramatically blocks the natural buoyancy force which reduces the overall heat transfer performance. But this phenomenon is not found at $R=0.15$ and 0.2. Although a positive rotation of the cylinder could restrain the natural convection flow, the enhancement of the flow intensity and better performance of heat transfer resulting from the forced convection compensates for the imposed restriction on natural convection.

The non-dimensional radius $(R)$ of the cylinder is another important parameter for the average Nusselt number. As for the motionless cylinder $(\Omega=0)$, increasing $R$ reduces the average Nusselt number. This is due to the cylinder pushing the fluid in the center toward the walls leading to the formation of a thinner thermal boundary layer at the heated and cooled walls and blocking the natural convection. It can be predicted that the maximum overall Nusselt number occurs at values between $R=0.05$ and $R=0.1$ from Fig. 8. Namely, there should be an optimum cylinder radius $R$, i.e., an optimum space between the cylinder and the walls, for a particular nanofluid to transfer heat and mass effectively as mentioned by Roslan et al. (2012). This is because moderate $R$ increased the disturbance of natural convection but did not block it. These results demonstrate transferring heat and mass can be effective where the forced convection becomes the dominant mechanism for fluid flow and for heat transfer. For instance, at $R=0.05$, the average Nusselt number decreases with increasing dimensionless rotational speed, and there

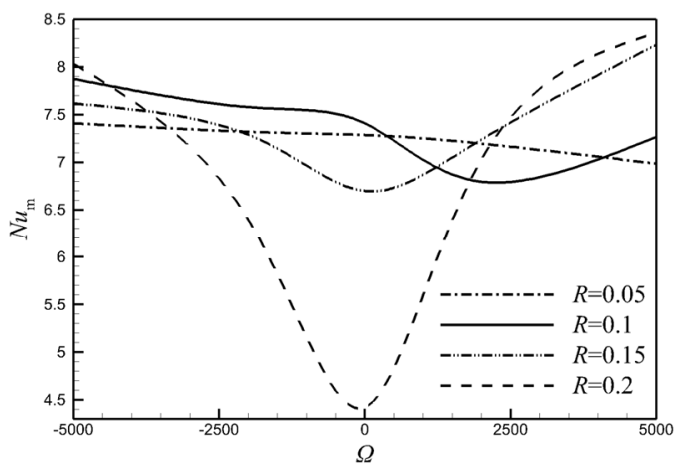

Fig. 8 Variation of average Nusselt number dependence on the dimensionless rotational velocity for different dimensionless radii at $\varphi=0.02$ and $R a=10^{5}$ is no pick up in that the intensification of imposed restrictions on natural convection gradually increases when $\Omega$ increases. However, the influence of the forced convection does not play an important role when the radius of the cylinder is comparatively small.

\subsection{Effects of nanoparticle volume fraction and the Rayleigh number}

Results of maintaining the rotating conditions and varying the nanoparticle concentration and the Rayleigh numbers are demonstrated in Table 3 and Figs. 9-11. It is assumed that the influence of the dimensionless rotation speed is not of interest in this part. The isotherm variation for different values of nanoparticle concentration and Rayleigh numbers are presented as the non-dimensional rotation speed $\Omega$ is 1000 and the non-dimensional radius of the inner cylinder $R$ tends to be 0.1 .

Table 3 The maximum value of the dimensionless stream function for different solid volume fraction and Rayleigh numbers at $\Omega=1000$ and $R=0.1$

\begin{tabular}{rrrrrr}
\hline \multirow{2}{*}{$R a$} & \multicolumn{5}{c}{ Dimensionless maximum stream function } \\
\cline { 2 - 6 } & \multicolumn{1}{c}{$\varphi=0$} & $\varphi=0.01$ & $\varphi=0.02$ & $\varphi=0.03$ & $\varphi=0.04$ \\
\hline $10^{4}$ & 1.0211 & 1.0113 & 0.9937 & 0.9652 & 0.8537 \\
$10^{5}$ & 8.7165 & 8.6669 & 8.4107 & 8.2723 & 7.9873 \\
$10^{6}$ & 26.7073 & 26.8381 & 26.9299 & 27.2159 & 27.2679 \\
$10^{7}$ & 44.9925 & 45.3513 & 45.4800 & 46.1239 & 46.2172 \\
\hline
\end{tabular}

The effects of the volume fraction of SiC nanoparticles on the flow of the nanofluids are quantified by a dimensionless maximum stream function as demonstrated in Table 3. For the selected range of nanoparticle concentrations, it is not obvious but still visible that the values of non-dimensional maximum stream functions in the cases of $R a=10^{4}$ and $R a=10^{5}$ decrease steadily as the solid volume fraction increases. The reason for this phenomenon is that the viscosity of SiC-EG nanofluids increases with an increase of the particle volume fraction as shown in Table 2. The increment of viscosity strengthens the viscous friction which leads to a drop of the flow intensity. However, a reverse behavior can be noticed at the cases of $R a=10^{6}$ and $R a=10^{7}$. At higher Rayleigh numbers, the natural convection becomes predominant and the additional viscous friction is negligible. 
Fig. 9 presents the isotherms at $\Omega=1000$ and $R=0.1$ where subfigures are scheduled going down with increasing volume fraction and going from left to right sides with increasing Rayleigh number. In general, the results obtained show that isotherms are strongly dependent on the Rayleigh numbers. For low Rayleigh numbers $\left(R a=10^{4}\right.$ and $\left.R a=10^{5}\right)$, the temperature contours are approximately parallel to each other at locations adjacent to the left hot surface, which indicates that the energy transport of the nanofluids is mainly conditioned by heat conduction. As the Rayleigh number increases, the role of convection in heat transfer becomes more significant and consequently the thickness of the thermal boundary layer near the wall decreases. As the Rayleigh number reaches $10^{7}$, almost all the temperature gradients are located in the interior of the boundary layers which have developed near the surfaces of the triangular enclosure. Moreover, the isotherms at the center of the cavity become completely horizontal, which indicates the heat transfer in the enclosure is mainly dominated by the convection state.
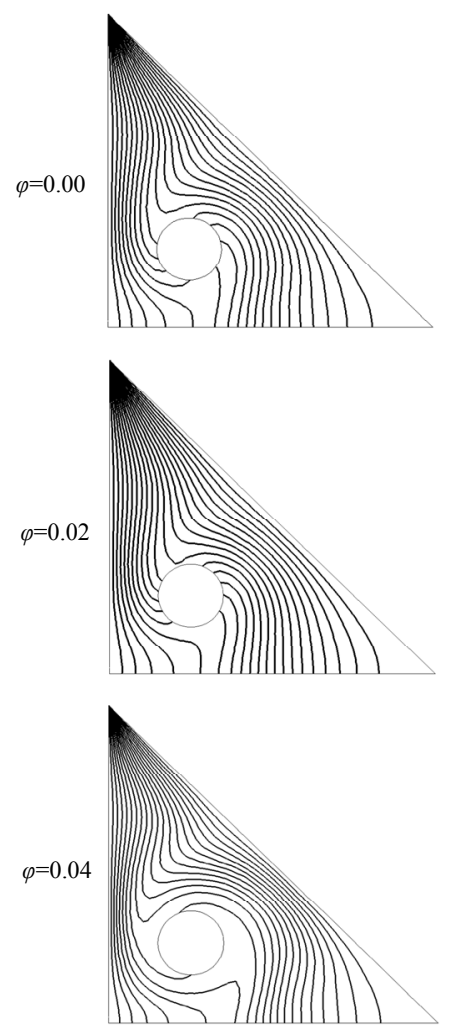

(a)
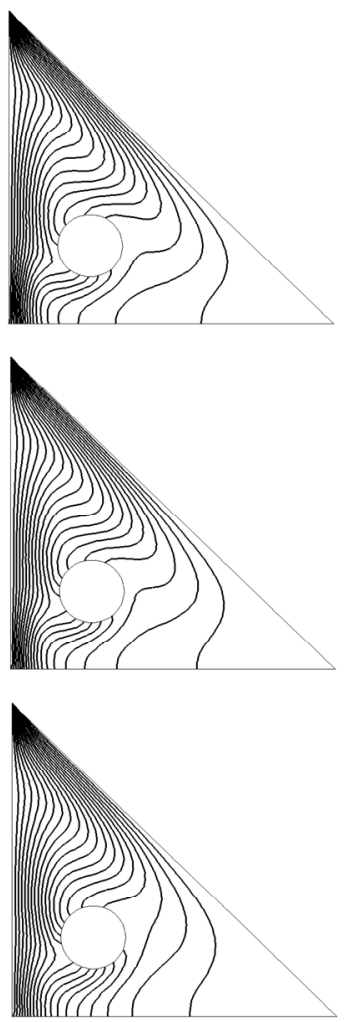

(b)
Further results are illustrated by the dimensionless velocity and temperature profiles at various Rayleigh numbers in Fig. 10. Note that the Rayleigh numbers affect the velocity and temperature profiles dramatically. In Figs. 10a and 10b, the variation in the vertical component of velocity $V$ and the dimensionless temperature $\Theta$ of the nanofluid flow, along the horizontal center line $(Y=0.5)$, are plotted. At high Rayleigh numbers, convection increases the energy exchange rates. The high peaks of the vertical component of velocity $V$ occur near the surfaces of the enclosure, and the slope of the dimensionless temperature profiles increases as the Rayleigh number increases. Figs. 10c and 10d present the horizontal component of the velocity $U$ and the dimensionless temperature $\Theta$, respectively. The velocity of nanofluids at high Rayleigh numbers is larger than that at lower ones, which enhances the flow intensity and causes greater convection and consequently results in an enhancement of the heat transfer. A check of the magnitude of $V$ and $\Theta$ at different Rayleigh numbers confirms the results obtained from analyzing
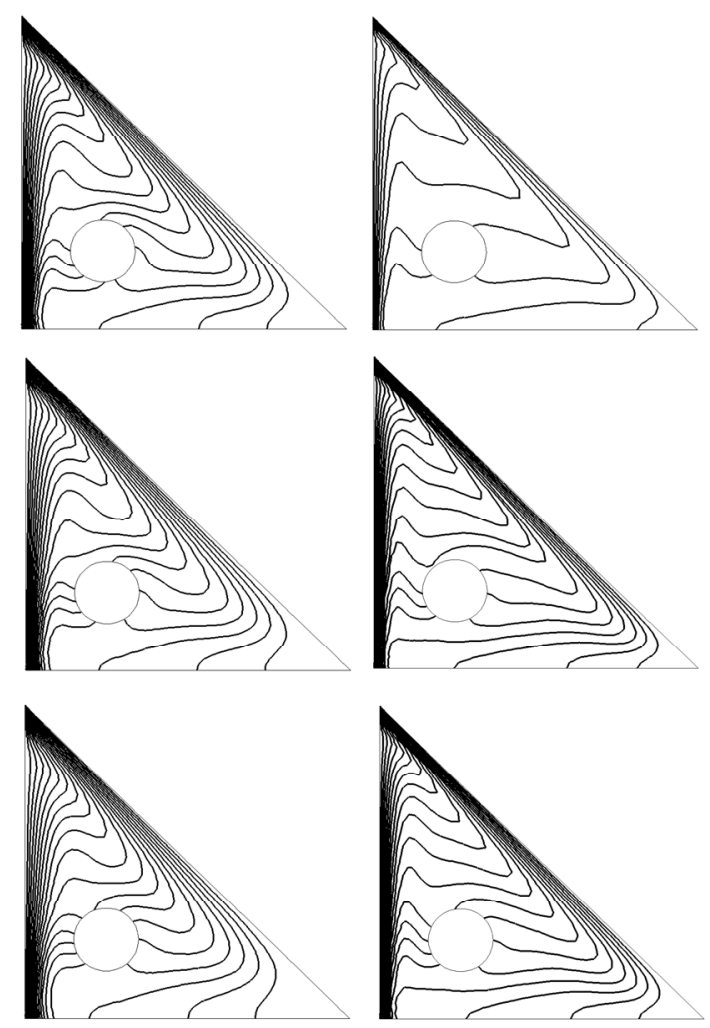

(c)

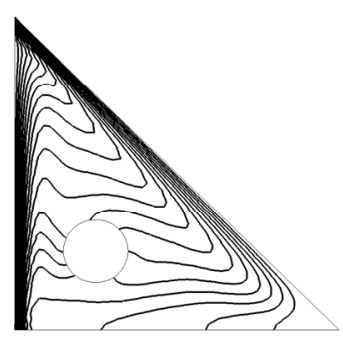

(d)

Fig. 9 Isotherms with respect to different volume fractions at $\Omega=1000$ and $R=0.1$ for $R a=10^{4}(a), R a=10^{5}(b), R a=10^{6}(c)$, and $R a=10^{7}(\mathrm{~d})$ 
the dimensionless maximum stream function and isotherms as shown previously in Table 3 and Fig. 10.

The heat transfer of $\mathrm{SiC}$ nanofluids is also examined by the average Nusselt number and the normalized Nusselt number. The results are depicted in Fig. 11a. First of all, it can be seen that the average Nusselt number increases with increasing Rayleigh number since the cases corresponding to high Rayleigh numbers involve a better thermal contact between the fluid and the isothermal surfaces than the cases with low Rayleigh numbers, and thus lead to a comparatively greater heat transfer crossing the enclosure. As expected, the heat transfer rate increases monotonically with the increase of the volume fraction of the $\mathrm{SiC}$ nanoparticles for all the Rayleigh numbers. It is worth pointing out that the value of thermal diffusivity becomes higher due to the presence of nanoparticles. In fact, as indicated by Soleimani et al. (2012), higher thermal conductivity is accompanied by higher values of thermal diffusivity as well, and the temperature gradients decrease at high thermal diffusivity. Accordingly, the boundary layer becomes thicker and this will in turn reduce the average Nusselt number. However, the effect of the slight drop in temperature gradient due to the presence of nanoparticles is insignificant compared to the increased thermal conductivity ratio, therefore the overall heat transfer performance is strengthened. Moreover, a normalized Nusselt number is defined as the ratio between surface averaged Nusselt numbers of SiC-EG nanofluids and pure EG as shown in Fig. 11b. The normalized Nusselt number is always the highest for the case of $R a=10^{4}$ for different solid volume fractions, which indicates that the most effective heat transfer is involved in this situation. This is mainly due to the following two factors: (1) As mentioned above, the heat transfer in the enclosure is predominantly by heat conduction at lower Rayleigh numbers and so is strongly dependent on the high thermal conductivity of nanofluids due to the presence of nanoparticles. Therefore, at lower Rayleigh numbers, the solid concentration becomes more effective on the enhancement of the heat transfer while, at high Rayleigh numbers, the process of heat transfer depends more on the buoyancy. (2) As forced convection starts to work, the heat transfer is enhanced, which was indicated by Misirlioglu (2006). Also, in this study, a lower Rayleigh number causes forced (a)

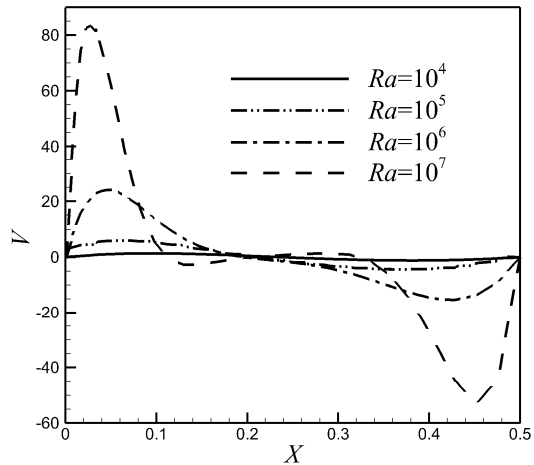

(b)

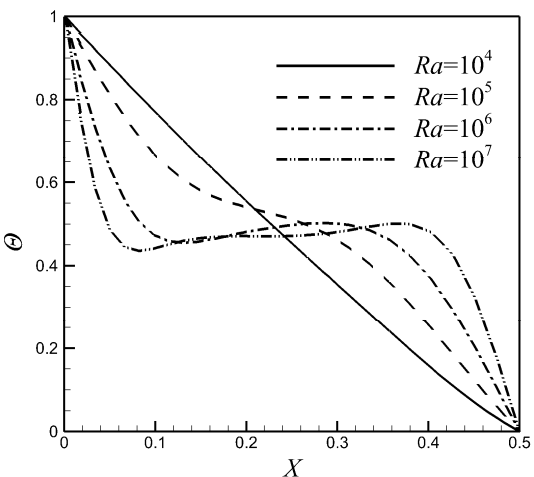

(c)

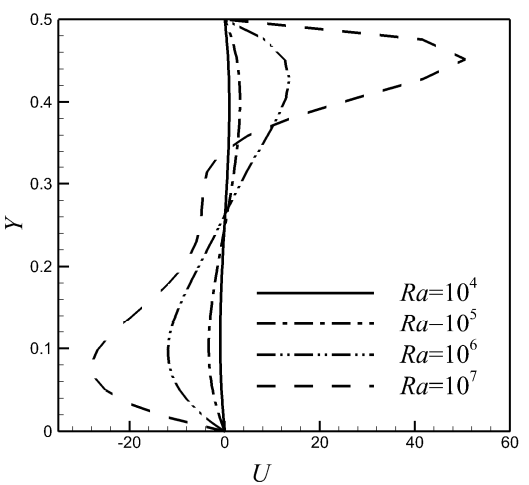

(d)

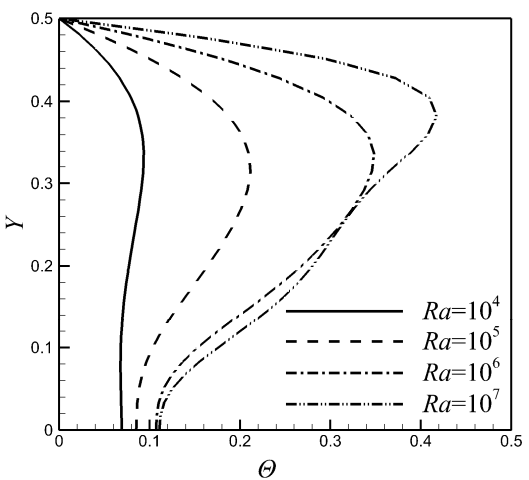

Fig. 10 Variation of dimensionless velocity $V$ at $Y=0.5$ (a) and $U$ at $X=0.5$ (c) and dimensionless temperature $\Theta$ at $Y=0.5$ (b) and $\Theta$ at $X=0.5$ (d) at different Rayleigh numbers at $\Omega=1000, R=0.2$, and $\varphi=0.02$ 

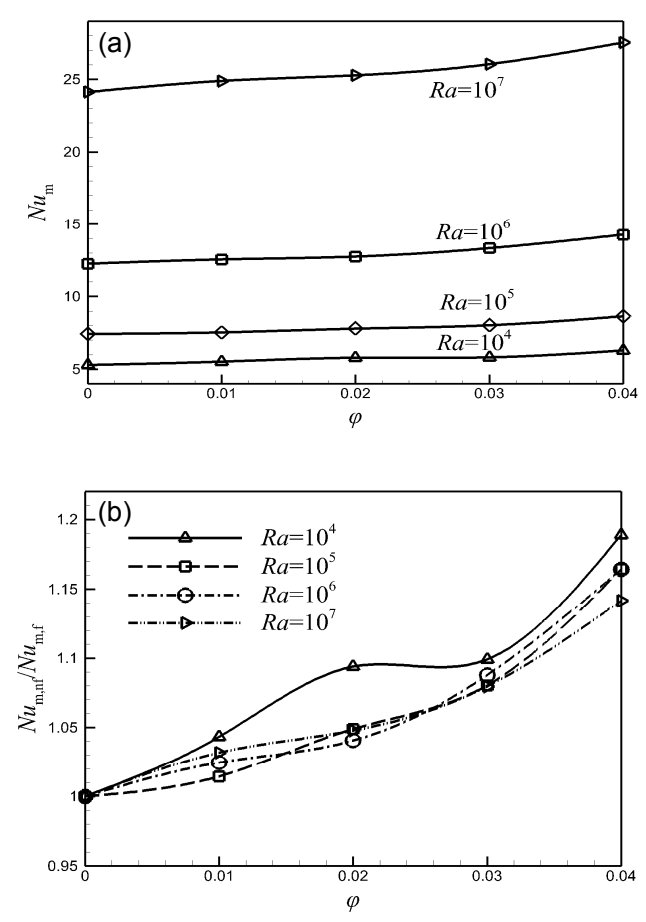

Fig. 11 Variation of average Nusselt number (a) and normalized Nusselt number $N u_{\text {m,nf }} / N u_{\text {m,f }}$ (b) with respect to the volume fraction of the nanoparticles for different Rayleigh numbers at $\Omega=1000$ and $R=0.1$

convection to dominate, which could explain why the normalized Nusselt number is always the highest for the case of $R a=10^{4}$.

\section{Conclusions}

The 2D steady-state laminar mixed convective flow and heat transfer around a horizontal rotational cylinder in a concentric triangular enclosure has been investigated numerically. Based on the results obtained, some concluding remarks can be summarized as follows:

1. Rotation of the inner cylinder has benefits for improving the heat transfer rate when the rotation direction is the same as the flow direction induced by natural convection. But the opposite rotational direction of the cylinder with lower rotational speed is prejudicial. Moreover, enhancement of heat transfer is obtained when forced convection becomes the dominant mechanism.

2. The heat transfer rate in the enclosure keeps increasing by adding $\mathrm{SiC}$ nanoparticles for a fixed dimensionless rotation speed and it is more pronounced at low Rayleigh numbers where heat conduction is more dominant.

3. It is observed that the size of the cylinder has a strong influence on the resulting flow and heat transfer process of SIC-EG nanofluids, as it limits the space for fluid flow between the cylinder and the enclosure walls. For high values of the cylinder radius, the average Nusselt number is small if the cylinder is almost motionless. On the other hand, to obtain better heat transfer performance in the triangular enclosure, negative rotation of the cylinder must be selected when the radius is smaller and for a motionless cylinder, an intermediate radius of cylinder must be chosen.

4. The strength of the flow circulation is always much stronger at $R=0.15$ for a cylinder with a faster rotation and at high Rayleigh numbers.

\section{Acknowledgements}

The authors would like to thank the Institute of Thermal Science and Power Systems, and the Department of Energy Engineering of Zhejiang University for their assistance in obtaining several important thermophysical properties (the thermal conductivity and viscosity of SiC-EG nanofluids).

\section{References}

Bararnia, H., Soleimani, S., Ganji, D.D., 2011. Lattice Boltzmann simulation of natural convection around a horizontal elliptic cylinder inside a square enclosure. International Communications in Heat and Mass Transfer, 38(10):1436-1442. [doi:10.1016/j.icheatmasstransfer.2011. 07.012]

Brinkman, H.C., 1952. The viscosity of concentrated suspensions and solutions. Journal of Chemical Physics, 20(4):571-581. [doi:10.1063/1.1700493]

Choi, S.U.S., Eastman, J.A., 1995. Enhancing thermal conductivity of fluids with nanoparticles. ASME International Mechanical Engineering Congress \& Exposition, San Francisco, USA.

Costa, V.A.F., Raimundo, A.M., 2010. Steady mixed convection in a differentially heated square enclosure with an active rotating circular cylinder. International Journal of Heat and Mass Transfer, 53(5-6):1208-1219. [doi:10. 1016/j.ijheatmasstransfer.2009.10.007]

Fu, W.S., Cheng, C.S., Shieh, W.J., 1994. Enhancement of natural convection heat transfer of an enclosure by a rotating circular cylinder. International Journal of Heat and Mass Transfer, 37(13):1885-1897. [doi:10.1016/00179310(94)90329-8]

Ghaddar, N.K., 1996. Natural convection over rotating cylindrical heat source in an enclosure. Journal of Thermophysics and Heat Transfer, 10(2):303-311. [doi:10.2514/ 3.788] 
Ghaddar, N.K., Thiele, F., 1994. Natural convection over a rotating cylindrical heat source in a rectangular enclosure. Numerical Heat Transfer, Part A: Applications: An International Journal of Computation and Methodology, 26(6):701-717. [doi:10.1080/10407789408956018]

Ghasemi, B., Aminossadati, S.M., 2010. Brownian motion of nanoparticles in a triangular enclosure with natural convection. International Journal of Thermal Sciences, 49(6): 931-940. [doi:10.1016/j.ijthermalsci.2009.12.017]

Kim, B.S., Lee, D.S., Ha, M.Y., et al., 2008. A numerical study of natural convection in a square enclosure with a circular cylinder at different vertical locations. International Journal of Heat and Mass Transfer, 51(7-8):1888-1906. [doi:10.1016/j.ijheatmasstransfer.2007.06.033]

Krane, R.J., Jessee, J., 1983. Some detailed field measurements for a natural convection flow in a vertical square enclosure. Proceedings of the First ASME-JSME Thermal Engineering Joint Conference, ASME, New York, 1:323-329.

Kuehn, T.H., Goldstein, R.J., 1976. An experimental and theoretical study of natural convection in the annulus between horizontal concentric cylinders. Journal of Fluid Mechanics, 74(04):695-719. [doi:10.1017/S0022112076 002012]

Lee, J.M., Ha, M.Y., Yoon, H.S., 2010. Natural convection in a square enclosure with a circular cylinder at different horizontal and diagonal locations. International Journal of Heat and Mass Transfer, 53(25-26):5905-5919. [doi:10. 1016/j.ijheatmasstransfer.2010.07.043]

Liao, C.C., Lin, C.A., 2012. Influences of a confined elliptic cylinder at different aspect ratios and inclinations on the laminar natural and mixed convection flows. International Journal of Heat and Mass Transfer, 55(23-24): 6638-6650. [doi:10.1016/j.ijheatmasstransfer.2012.06.073]

Maxwell, J.C., 1904. A Treatise on Electricity and Magnetism. 2nd Edition, Oxford University Press, Cambridge, p.435-441.

Misirlioglu, A., 2006. The effect of rotating cylinder on the heat transfer in a square cavity filled with porous medium. International Journal of Engineering Science, 44(18-19): 1173-1187. [doi:10.1016/j.ijengsci.2006.07.008]

Moukalled, F., Acharya, S., 1996. Natural convection in the annulus between concentric horizontal circular and square cylinders. Journal of Thermophysics and Heat Transfer, 10(3):524-531. [doi:10.2514/3.820]

Parvin, S., Alim, M.A., Hossain, N.F., 2012. Prandtl number effect on cooling performance of a heated cylinder in an enclosure filled with nanofluids. International Coтmunications in Heat and Mass Transfer, 39(8):1220-1225. [doi:10.1016/j.icheatmasstransfer.2012.06.006]

Roslan, R., Saleh, H., Hashim, I., 2012. Effect of rotating cylinder on heat transfer in a square enclosure filled with nanofluids. International Journal of Heat and Mass Transfer, 55(23-24):7247-7256. [doi:10.1016/j. ijheatmasstransfer.2012.07.051]
Shih, Y., Khodadadi, J., Weng, K., et al., 2009. Periodic fluid flow and heat transfer in a square cavity due to an insulated or isothermal rotating cylinder. Journal of Heat Transfer, 131(11):111701. [doi:10.1115/1.3154620]

Soleimani, S., Sheikholeslami, M., Ganji, D.D., et al., 2012. Natural convection heat transfer in a nanofluid filled semi-annulus enclosure. International Communications in Heat and Mass Transfer, 39(4):565-574. [doi:10.1016/ j.icheatmasstransfer.2012.01.016]

Xu, X., Yu, Z.T., Hu, Y.C., et al., 2010. A numerical study of laminar natural convective heat transfer around a horizontal cylinder inside a concentric air-filled triangular enclosure. International Journal of Heat and Mass Transfer, 53(1-3): 345-355. [doi:10.1016/j.ijheatmasstransfer.2009.09.023]

Xu, X., Yu, Z.T., Hu, Y.C., et al., 2012. Transient natural convective heat transfer of a low-Prandtl-number fluid from a heated horizontal circular cylinder to its coaxial triangular enclosure. International Journal of Heat and Mass Transfer, 55(4):995-1003. [doi:10.1016/j.ijheatmasstransfer. 2011.10.011]

Yu, Z.T., Xu, X., Hu, Y.C., et al., 2010. Transient natural convective heat transfer from a heated triangular cylinder to its air-filled coaxial cylindrical enclosure. International Journal of Heat and Mass Transfer, 53(19-20):4296-4303. [doi:10.1016/j.ijheatmasstransfer.2010.05.058]

\section{中文概要}

\section{题 目:三角形封闭腔内内含旋转柱体时碳化硅-乙二醇纳} 米流体层流混合对流传热特性研究

目 的: 明确在封闭腔内放置旋转柱体时, 柱体尺寸及转 速对不同浓度下纳米流体的流动传热的影响。

创新点: 1. 数值模拟中采用的碳化硅-乙二醇 ( $\mathrm{SiC}-\mathrm{EG}$ ) 纳米流体的重要热物性参数均为实验测量值; 2. 考虑封闭腔内柱体的动态旋转对腔内纳米流 体流动传热的影响。

方 法: 基于对 $\mathrm{SiC}-\mathrm{EG}$ 纳米流体导热系数与粘度的实验 测量, 采用数值模拟方法探究封闭腔内旋转柱 体、纳米流体浓度以及瑞利数对 $\mathrm{SiC}-\mathrm{EG}$ 纳米流 体流动传热性能的影响。

结 论: 1. 在柱体的旋转方向与由自然对流引起的纳米流 体流动方向相同的情况下, 置于腔内的旋转柱体 可以起到强化传热的效果。2.二者旋转方向相反 时情况较为复杂, 当柱体尺寸较小且柱体转速较 低时削弱传热效果; 当柱体尺寸较大且转速较高 而引发的强制对流占主导地位时, 将对腔内传热 起到一定的强化效果。

关键词: SiC-EG 纳米流体; 混合对流; 三角封闭腔; 旋转 柱体; 瑞利数 